\title{
FILODEMO Y LA PARRHĒSÍA EPICÚREA COMO PRÁCTICA PEDAGÓGICO-TERAPÉUTICA
}

\author{
PHILODEMUS AND EPICUREAN PARRHESSÍA \\ AS A PEDAGOGICAL-THERAPEUTIC PRACTICE \\ Rodrigo Braicovich
}

\begin{abstract}
RESUMEN
El objetivo del escrito consiste en analizar ciertas objeciones que han sido realizadas recientemente a lo que denominaré la Interpretación pedagógica del Perí Parrhèsías de Filodemo de Gadara. Luego de reconstruir brevemente dicha interpretación y de pasar revista a las objeciones que han sido formuladas, presentaré las razones por las que considero que (una versión modificada y ampliada de) la interpretación pedagógica todavía representa el modelo más adecuado de abordaje del tratado.
\end{abstract}

PALABRAS ClAVE: epicureísmo; parrhèsía; pedagogía; psicagogía.

\begin{abstract}
The aim of the paper is to analyze certain objections against what I will call the Pedagogical Interpretation of Philodemus of Gadara's Perí Parrhèsía. After presenting a brief summary of that interpretation and reviewing the objections that have recently been raised, I will present the reasons why I consider that (a modified and expanded version of) the Pedagogical Interpretation still represents the most adequate approach to the treatise.
\end{abstract}

KEY WORDS: Epicureanism; parrhēsía; pedagogy; psychagogy.

\section{INTRODUCCIÓN}

A pesar de que no pretende ser más que un epítome de las lecciones orales brindadas por su maestro Zenón de Sidón, el tratado de Filodemo de Gadara titulado Peri parrbēsias [de aquí en adelante, PP] ha despertado, desde su primera publicación en 1835, el interés de numerosos comentaristas, y esto por dos motivos fundamentales: en primer lugar, porque, a pesar de su estado 
sumamente fragmentario, representa uno de los escasos tratados antiguos que se han conservado dedicado a la problemática específica de la parrbèsía, un concepto que ha sido interpretado como un elemento central de la política democrática clásica $^{1}$. En segundo lugar, porque nos permite reconstruir (al menos según lo que denominaré, en las secciones siguientes, la interpretación pedagógica) una práctica terapéutica específica, la cual representaría un elemento esencial en el repertorio psicagógico de la comunidad epicúrea. El estado de preservación del PP ha representado, no obstante, un obstáculo decisivo en el camino hacia la reconstrucción del sentido que asumía la parrhèsía en el interior de las comunidades epicúreas, y los interrogantes que se han suscitado en las últimas décadas entre los comentaristas han contribuido a volver sumamente complejo un panorama que en sus inicios parecía relativamente simple: ¿es la parrhèsía descripta por Filodemo ${ }^{2}$ una práctica específicamente pedagógica o se trata, por el contrario, de una práctica compartida por todo ateniense o romano libre que el epicureísmo incorpora en su seno e intenta enmarcar dentro de ciertos límites éticos? ¿Estamos ante una práctica propia de las comunidades epicúreas desde su origen, o remite a una innovación propia del período italiano de la escuela? ¿Cómo se articula la parrhésía filodemea con psicología epicúrea o, más específicamente, con la teoría epicúrea de la acción? A partir del ya clásico estudio sistemático de Arnaldo Momigliano sobre la historia de la libertad de expresión en la antigüedad clásica, ha devenido un lugar común señalar que con la caída de la polis y el comienzo del período helenístico, la parrhèsía deviene una excelencia propia del filósofo y deja de ser un derecho político para pasar a ser una virtud privada ${ }^{3}$. Como señala Holland para el caso específico del epicureísmo: "The political virtue once exercised in the public forum has become

\footnotetext{
${ }^{1}$ Aunque solo indirectamente vinculados con el problema de la parrhèsía, se han conservado, además del PP, un tratado de Plutarco (De adulatore) y un diálogo de Luciano de Samóstata (Revivescentes sive piscator).

${ }^{2}$ Aun cuando el PP se presente como una epítome de las lecciones orales de Zenón, omitiré en las siguientes páginas, meramente por cuestiones prácticas, toda nueva aclaración al respecto, dejando a un lado el problema — en última instancia irresoluble — de distinguir entre la voz de Zenón y la de Filodemo.

${ }^{3}$ Cf. Momigliano 260. Los antecedentes previos al epicureísmo en el ámbito específicamente filosófico no son demasiados: un puñado de pasajes platónicos aislados (Rep. 557b; Symp. 222c, Phdr. 240d), la consideración de Aristóteles de la parrhèsía como parte de la megalopsychia (EN 1124b29), y la caracterización, por parte de Diógenes de Sínope, de la parrhèsía como "lo más hermoso entre los hombres" (DL 6.69). Respecto de la compleja relación entre parrhèsía e ironía en los diálogos socráticos, cf. Erler 2011a.
} 
a personal virtue to be practiced primarily within the confines of the Epicurean community" (Holland 2004, pp. 256-257). Nada de esto, no obstante, nos indica el lugar específico que la parrhèsía puede haber asumido en el interior de la comunidad epicúrea. Nada nos dice, fundamentalmente, respecto de la pregunta acerca de si la misma fue concebida simplemente como una excelencia del carácter, una virtud que todo individuo racional debe aspirar a conquistar, o si devino una técnica terapéutica específica, articulada con los objetivos generales de la psicagogía epicúrea. Esto es lo que, al menos de modo parcial, intentaré responder en las siguientes páginas, evaluando dos alternativas de lectura marcadamente opuestas: la interpretación pedagógica, por un lado, y la lectura defendida recientemente por Julie Giovacchini, quien ha propuesto una relectura radical del fenómeno de la parrhêsía epicúrea. Como intentaré demostrar en la cuarta sección, aun cuando las objeciones de Giovacchini a la interpretación pedagógica poseen inicialmente cierta plausibilidad, existen razones importantes para defender de dicha interpretación, o, cuanto menos, una versión reformada y ampliada de la misma.

\section{EL STATUS DE LA PARRHĒSÍA EN LA INTERPRETACIÓN PEDAGÓGICA}

¿Cuál es el status que asignaban los epicúreos a la parrbēsía? ¿Qué lugar asumía dentro de la dinámica pedagógica que se establecía en el interior de la comunidad epicúrea entre maestros y discípulos? ¿Representaba meramente una práctica frecuente y heredada de una tradición anterior y externa a la escuela, o era considerada, por el contrario, como un dispositivo pedagógico-terapéutico específico? La línea de lectura más difundida en las últimas décadas (la cual toma su origen fundamentalmente en la interpretación propuesta hacia 1970 por Marcello Gigante $^{4}$ y adquiere amplia difusión a través de la publicación de las lecciones de dictadas por Michel Foucault durante 1981-19825) ha sostenido, en forma tajante, que sí: la parrbèsía epicúrea constituye, de acuerdo a dicha línea de interpretación, una práctica claramente reglada e institucionalizada, de carácter estocásticoconjetural, que tiene por objetivo — en claro paralelo con el arte médica— curar el alma del alumno a través de la palabra, del discurso libre y franco. Desde el punto de vista historiográfico, esta institucionalización de la parrhêsía como una práctica pedagógica específica representaría una innovación decisiva respecto de la

\footnotetext{
${ }^{4}$ Cf. Gigante 1975; 1983.

${ }^{5}$ Cf., a modo de ejemplo, Foucault 2001; Tsouna 2007; Glad 2010, Konstan et al.; Erler 2011b; Nussbaum 1996, pp. 133-135; Fernandes.
} 
tradición anterior (analogable, por su carácter institucionalizado y explícitamente reglado, por ejemplo, a la confesión cristiana ${ }^{6}$ ), una creación propia del epicureísmo que habría tomado cuerpo en los inicios de la escuela y se habría desarrollado y pulido en forma sistemática a lo largo de, cuanto menos, los dos siglos siguientes. Hasta el surgimiento de la comunidad epicúrea, en efecto, el concepto de parrhésía habría representado la práctica tradicional (dentro de la cultura media de las clases atenienses cívica y políticamente formadas), pero informaly desregulada, de hablar con franqueza, veracidad y libertad frente a cualquier interlocutor. El desplazamiento mediante el cual la parrhèsía deja de representar un "derecho político" para devenir una "virtud privada" excede, desde ya, al epicureísmo, pero es en el interior de dicha escuela en donde la parrbèsía habría adquirido un estatuto específico como dispositivo pedagógico-psicagógico, un dispositivo que no solo formaba parte del repertorio pedagógico del maestro epicúreo, sino que representaba la herramienta pedagógica esencial. Voula Tsouna, quien ha ofrecido la reconstrucción más sistemática hasta el momento de la dinámica de la parrbèsía en el interior de la comunidad epicúrea, resalta precisamente la centralidad de dicha práctica:

"Parrbesía is a central feature of the Epicurean education and the Epicurean way of life. As a pedagogical method, it is flexible and nuanced, and has effective ways of engaging the students' entire personality in order to achieve their moral improvement. It is applied according to sound educational principles and incorporates astute psychological insights. More broadly conceived, as a way of speaking, parrhessia determines the nature of the Epicurean communities and the relationships between their members. It accounts to a large extent for the cohesion of these communities, the transmission of Epicureanism from the teachers to the students, and the systematic promotion of Epicurean values and goals." (Tsouna 2007, p. 118)

En líneas generales, la interpretación pedagógica puede ser sintetizada definiendo la parrhèsía como:

a) una práctica pedagógico-psicagógica,

b) de tipo estocástica-conjetural,

c) construida sobre una dinámica "rotativa",

d) explícitamente reglada,

e) apelando a la confesión voluntaria por parte del alumno o, alternativamente, a la delación amigable por parte de sus compañeros.

${ }^{6}$ Cf, vg. Nussbaum 1996, pp. 133-134. 
Antes de analizar la segunda línea de lectura, conviene desarrollar brevemente algunos de los últimos puntos (dado que el primero representa, en cierta medida, el corolario de los restantes). Respecto del segundo punto: ¿qué significa la caracterización de la parrhèsía como una práctica o un arte (technêe) de tipo estocásticoconjetural? Fundamentalmente, que constituye una práctica que es plenamente consciente de la imposibilidad de definir una metodología de tipo universal, es decir, una serie de reglas generales que sean válidas para todos los escenarios posibles que el maestro deba enfrentar ${ }^{7}$. Esto trae aparejada una segunda característica, que consiste en el hecho de que la parrhèsía aparece como una práctica igualmente consciente de la imposibilidad lógica de asegurar su propio éxito (dada, por un lado, la falibilidad propia del ser humano y, por otro, la presencia en la ecuación pedagógica de elementos que no dependen del maestro). En este sentido, contra la lectura defendida por Martha Nussbaum, quien propone interpretar la psicacogíapedagogía helenística bajo el modelo de relaciones jerárquicas claramente marcadas y rígidas, Clarence Glad ha enfatizado precisamente el hecho de que, al menos en el caso de la parrbèsía, el recurso por parte de Filodemo al discurso médico no tiene por objetivo definir una relación jerárquica entre alumno y docente (médico y paciente), sino, por el contrario, enfatizar el carácter conjetural de la parrbèsía: en primer lugar, así como el médico debe atender a la especificidad de la enfermedad, del paciente y del contexto en el cual éste se halla inserto, así también el parresiasta debe tomar en cuenta la situación específica de su interlocutor, su posición social, su sexo, su educación, su formación filosófica, etc. Más aún: tal como el médico puede eventualmente errar su diagnóstico o, aun habiendo realizado una evaluación adecuada de la situación, no lograr la recuperación del paciente, lo mismo puede suceder en el caso de la parrhèsía.

El tercer punto otorga apoyo adicional al reclamo de Glad: si la parrhèsía epicúrea se construyera sobre una dinámica jerárquica, carecerían de sentido las numerosas referencias que encontramos en el PP a lo que Konstan y Glad han denominado una "psicagogía rotativa" (Konstan et al. 24; Glad 2010, p. 124), esto es, una práctica en la cual los polos de la relación pueden verse invertidos en cualquier momento, y aquél que ejercía hace un momento la parrhèsía es ahora

\footnotetext{
${ }^{7}$ De allí que la construcción de una especie de repertorio tipológico que ofrece el PP (definido en función de la mayor o menor permeabilidad de cada carácter —el joven, el viejo, la mujer, etc.- a la parrhèsía; cf. Cols. 22a-24a) se vea contrapesado con la advertencia respecto de la posibilidad de que aun el sabio epicúreo cometa, por distintas razones, errores — comprensibles y perdonables — en su diagnóstico del alumno (cf., paradigmáticamente, Fr. 47).
} 
quien va a ser interpelado, ya sea por su interlocutor previo, o por otro integrante de la comunidad ${ }^{8}$. La importancia de este elemento (como veremos en la sección cuarta) radica en el hecho de que habilita la parrhèsía como una práctica que puede tener lugar no solo (a) entre un maestro y un alumno, sino también (b) entre dos alumnos o aun (c) entre dos maestros/sabios.

Respecto del cuarto punto, la idea de que estamos ante una práctica explícitamente reglada se apoya en el hecho de que el $P P$ representa, precisamente, un especie de manual de uso para el parresiasta, en el cual éste puede encontrar definidas con precisión las reglas específicas que rigen la práctica de la parrbèsía en el interior de la comunidad epicúrea. Allí encontramos, a modo de ejemplo, indicaciones respecto del momento oportuno (kairós) para hablar francamente con el alumno, enfrente de quiénes la amonestación debe tener lugar, o en qué casos la intervención del maestro se encuentra justificada. El último punto atañe a dos elementos al que algunos defensores de la interpretación pedagógica han otorgado una clara centralidad en la dinámica de la parrhèsía: por un lado, la confesión voluntaria por parte del individuo que ha cometido una falta,; por otro, la delación, por parte de terceros, de las faltas percibidas en el otro, denuncia motivada por una preocupación genuina por el bienestar de aquél a quien se está denunciando ante el maestro para ser corregido e instruido. El apoyo textual que el $P P$ ofrece para cada una de estos dos elementos, es ciertamente dispar: mientras que la confesión voluntaria se halla claramente atestiguada en numerosos fragmentos ${ }^{9}$, y aparece como un momento necesario en el desarrollo de la parrhèsía, la figura de la delación bienintencionada se apoya en un conjunto mucho más reducido de fragmentos ${ }^{10}$, lo cual vuelve hasta cierto punto cuestionable el lugar central que ciertos autores le han otorgado en la dinámica de la parrhèsía.

\section{LAS OBJECIONES DE GIOVACCHINI A LA INTERPRETACIÓN PEDAGÓGICA}

Como se hace evidente por lo señalado hasta aquí, la existencia del PP de Filodemo es absolutamente fundamental para la interpretación pedagógica, dado

\footnotetext{
${ }^{8}$ Desde esta perspectiva, hablar de la parrhèsía, como venimos haciendo hasta el momento, como una relación que se establece entre "maestro" y "alumno" representa una caracterización claramente imprecisa. Aun así, seguiré utilizando ambos términos por una cuestión meramente práctica y por falta de un binomio conceptual que exprese con mayor precisión las posiciones que se hallan en juego.

${ }^{9}$ Vid., entre otros, Fr. 41, 42, 49, 51, 55, 75, 76.

${ }^{10}$ Vid. Fr. 50, 52, 53, 76.
} 
que la totalidad de los elementos que señalé anteriormente como centrales para dicha interpretación depende de pasajes y secciones específicas de dicho tratado. Según ha sugerido recientemente Julie Giovacchini, no obstante, la mera existencia de dicho tratado no garantiza por sí misma la validez de la interpretación pedagógica, dado que esta última misma depende, según la autora, de un ordenamiento específico de los fragmentos que se han conservado del texto.

Para entender el núcleo de la objeción de la autora, es preciso tener en cuenta el estado absolutamente fragmentario del texto que poseemos en la actualidad, el cual se halla compuesto por 94 fragmentos y 24 columnas — cuya sección intermedia se ha perdido irremediablemente — reconstruidos a partir de papiros recuperados en la "Villa dei Papiri" de la ciudad romana de Herculano. Desde el punto de vista hermenéutico, las dificultades más importantes que enfrentan tanto el editor como el lector son las siguientes: i) dificultad para articular temáticamente las partes superior e inferior de las columnas preservadas; ii) dificultad para comprender la conexión temática entre una columna y la siguiente; iii) dificultad o imposibilidad de articular entre sí temáticamente los fragmentos preservados ${ }^{11}$. Aun cuando la editio princeps de los fragmentos fue publicada en 1835 y 1843 (en Voluminum Herculanensium V), la edición que ha servido de base a las ediciones posteriores y los estudios críticos ha sido la realizada por Alexander Olivieri para Teubner en 1914 (incluyendo la edición crítica más reciente del tratado, a saber, la edición bilingüe griego-inglés realizada en 1998 por David Konstan et al para la Society of Biblical Literature $)^{12}$. Lo relevante del caso es el hecho de que el ordenamiento de las columnas y fragmentos propuesto por Olivieri, ordenamiento que ha servido de base a la gran mayoría de las interpretaciones contemporáneas del tratado, no sólo se encuentra lejos de ser incuestionable, como Konstan et al. admiten ${ }^{13}$, sino que, en rigor, no se encuentra fundamentado en ningún criterio temático, dado que Olivieri se habría limitado a disponer los fragmentos siguiendo el orden en el que los mismos fueron enmarcados por los trabajadores manuales encargados de separar mecánicamente las capas de los rollos. Al menos en el caso de los

${ }^{11}$ White señala, a este respecto, dos ordenamientos posibles: o bien suponemos que los fragmentos preservados componen (con lagunas) un texto continuo, o bien que representan porciones superiores o inferiores de columnas distanciadas entre sí (cf. White 121-122). Como se hace evidente, el resultado que arrojaría cada uno de los dos agrupamientos podría diferir sustancialmente respecto del otro.

${ }^{12}$ Para una descripción detallada de las condiciones de reconstrucción del papiro, vid. White.

${ }^{13}$ Konstan et al. 10. 
fragmentos, entonces, el orden (verdaderamente "desconcertante", en palabras de Delattre $^{14}$ ) en el que los fragmentos han sido presentados en las ediciones desde Olivieri en adelante no obedece a criterio temático, lógico o cronológico alguno. Tomando como ejemplo la supuesta práctica epicúrea de la delación de los pares, Giovacchini ha enfatizado hasta qué punto un re-ordenamiento de los fragmentos puede alterar sustancialmente la centralidad que asignamos a un determinado elemento y reconfigurar, con ello, nuestra lectura de la parrhésía epicúrea:

“Etant donné que des allusions à la délation bienveillante - c'est-à-dire au fait pour un élève d'en dénoncer un autre auprès des maîtres «pour son bien» - apparaissent dans plusieurs cadres, et donc en des endroits du texte proposé par Olivieri plutôt éloignés les uns des autres, les interprètes ont pu avoir l'impression qu'il s'agissait là d'une pratique particulièrement fréquente et, de ce fait, importante et valorisée par l'épicurisme. La reconstruction bibliologique montre aujourd'hui que ce n'est pas vraiment le cas, puisque dans le rouleau nouvellement reconstruit, les allusions à la délation bienveillante se trouvent, pour l'essentiel, concentrées dans une seule et unique séquence constituée par les fr. 49-54 et 79 N-80 O., soit les col. 156-161 D." (Giovacchini 305)

En función de las dificultades implícitas en la interpretación pedagógica, Giovacchini ha propuesto una relectura alternativa del tratado, la cual ataca directamente la interpretación de la parrhèsía epicúrea como una práctica pedagógica específica y sugiere, por el contrario, entender el PP como un tratado dirigido fundamentalmente a establecer las condiciones bajo las cuales la práctica de la parrbésía sería legítima ${ }^{15}$. Las críticas de la autora a la interpretación pedagógica, y la lectura que propone como alternativa, pueden ser resumidas como sigue:

${ }^{14}$ Cf. Delattre 438.

${ }^{15}$ La interpretación pedagógica y la de Giovacchini no agotan, desde ya, el campo de lecturas posibles. Algunos comentaristas han optado, a modo de ejemplo, por enmarcar la parrhèsía epicúrea en el contexto del problema de la philía y en oposición al fenómeno de la adulación, lo cual permitiría reinsertar la perspectiva epicúrea en la tradición tanto precedente como posterior a Filodemo. Si bien la triple articulación philía-parrhèsía-kolakeia representa ciertamente una perspectiva a tener en cuenta al abordar tanto el PP como su relación con el Peri kolakeias, una lectura mínima de aquél hace evidente que el tratamiento filodemeo no sólo no se agota en el diálogo entre franqueza y adulación, sino que dicha contraposición se muestra como claramente secundaria. Cabe señalar, es cierto, que los autores que han privilegiado dicho enfoque no han pretendido ofrecer una lectura alternativa a la interpretación pedagógica (como hace Giovacchini), sino simplemente una perspectiva diferente de ingreso al texto. Cf., entre otros, Kemp, Glad 1996, Konstan 1996. 
1. Fuera del PP, no existe registro alguno que testifique la existencia de una práctica específicamente epicúrea de la parrbèsía: ninguna de las tres instancias que se han conservado, fuera del tratado, del término parrhēsía (o de sus posibles equivalentes latinos) apunta a otra cosa que a la mera virtud privada, ya clásica y en absoluto específica del ideal epicúreo, de hablar con franqueza o sinceridad ${ }^{16}$.

2. Respecto de la caracterización de la parrhēsía como una técnica estocástica (techne stochastikêe), la evidencia es virtualmente nula: la mera caracterización de la acción del sabio como stochazomenos (limitada al Fr. 1) no es suficiente para compensar la ausencia total de referencias en el tratado al concepto de $\operatorname{techne} e^{-17}$.

3. En cuanto al estatus de la parrhèsía invocada en el tratado, nada permite suponer que sea otra cosa que la mera práctica, tan frecuente dentro como fuera de los círculos epicúreos, de hablar con franqueza ante aquellos por quienes nos preocupamos —una práctica que, no obstante, exige ser realizada siguiendo un conjunto de reglas deontológicas ${ }^{18}$.

4. El objetivo del tratado, en consecuencia, no es exponer las condiciones bajo las cuales la parrbēsía deviene una práctica pedagógica exitosa, sino únicamente presentar las reglas que guían la práctica correcta o éticamente aceptable de la parrhēesía como interacción, no específicamente pedagógica ni aun filosófica, entre dos individuos. El resultado al que apuntaría la implementación de dichas reglas, en este sentido, consistiría primordialmente en desterrar prácticas ilegítimas y abusivas que, bajo la pretensión de preocuparse por el otro, dan rienda suelta a actitudes ciegas y violentas o, cuanto menos, actitudes que desatienden el contexto en el cual se enmarca la acción del interlocutor ${ }^{19}$.

Las consecuencias de la propuesta de Giovacchini son radicales, como se hace evidente, en la medida en que despoja a la parrbèsía de todos los elementos que la dotaban —en el interior de la interpretación pedagógica- de interés terapéutico y la volvían una innovación propia del epicureísmo. La parrbèsía, de esta forma, se

\footnotetext{
${ }^{16}$ Cf. Giovacchini 295-296.

${ }^{17}$ Cf. Giovacchini 297-300.

${ }^{18}$ Daniel Delattre señala, en un sentido análogo, que el contexto adecuado para comprender el $P P$ es el de un diálogo con la tradición cínico-estoica, una tradición que, según Delattre, Filodemo no puede haber ignorado al componer el tratado. Vid. Delattre 437.

${ }^{19}$ Cf. Giovacchini 308-311.
} 
ve expulsada del repertorio de prácticas psicagógicas específicas, en cuyo marco había sido interpretada por la lectura pedagógica, y el tratado de Filodemo deviene un mero manual de uso para los integrantes de la comunidad epicúrea, reintegrado, nuevamente, a una tradición que será continuada, entre otros, por Plutarco (en De adulatore), y que poco (o nada) posee de específicamente epicúreo.

\section{EL PROBLEMA DE LA EFICACIA PEDAGÓGICA DE LA PARRHÉSÍA}

Las objeciones de Giovacchini a la interpretación pedagógica representan una advertencia saludable, que puede ser pensada en el marco de lo que —en otra ocasión- he enfatizado como una tendencia a interpretar ciertas prácticas ocasionales dentro de las escuelas helenísticas en términos excesivamente institucionales, es decir, a atribuir un carácter sistemático, reglado y consciente a ciertos actos, hábitos o prácticas que no representan más que actos espontáneos al que los filósofos clásicos y helenísticos quizás no atribuían ninguna dimensión filosófica o pedagógica específica ${ }^{20}$. Aun cuando esta tendencia parece exacerbarse entre los comentaristas contemporáneos fundamentalmente desde la obra de Pierre Hadot en adelante, el ejemplo más paradigmático de esta tendencia, en el caso puntual del epicureísmo, podemos encontrarlo en la obra de Norman De Witt, particularmente en su célebre "Organization and procedure in Epicurean groups" (De Witt 1936), en donde el autor ofrece una imagen de la dinámica interna de las comunidades epicúreas rígidamente estructurada y jerarquizada, y, desde el punto de vista pedagógico, minuciosamente planificada ${ }^{21}$. Las objeciones de Giovacchini, en este sentido, pretenden ponernos sobre alerta respecto de los peligros latentes al momento de rellenar las lagunas y componer cuadros de conjunto de las escuelas helenísticas (en este caso) a partir de fragmentos, lagunas y silencios.

El diagnóstico general de la autora, no obstante, merece ser revisado, revisión que concierne no solo al análisis específico que realiza respecto del $P P$, sino también (y de modo aún más fundamental) a la conclusión global que deriva de dicho análisis. La interpretación de la autora propone, en líneas generales, dos frentes de ataque a la lectura pedagógica: por un lado, reclamando la nulidad de algunos de los elementos que componen dicha lectura (la delación amigable, el carácter rotativo de la parrbèsía, y la caracterización de la misma como técnica

\footnotetext{
${ }^{20}$ Vid. Braicovich.

${ }^{21}$ Ver asimismo De Witt 1937 y 1954.
} 
estocástica); por otro, destacando los puntos de continuidad entre el tratado de Filodemo y la tradición anterior. A partir de la conjunción de ambos frentes de crítica, la autora deriva la negación radical de toda dimensión específicamente epicúrea en el tratamiento filodemeo de la parrhèsía:

"Ce livre peut ainsi être envisagé soit comme une vigoureuse défense du Jardin dirigée contre ceux qui accusent les épicuriens de ne pas pratiquer correctement le franc-parler, soit comme un manuel de bonnes pratiques à l'usage de ceux qui fréquentent le Jardin, soit encore comme un mixte des deux; mais il ne faut, probablement, rien y chercher de plus." (Giovacchini311)

¿Es la conclusión de Giovacchini correcta? ¿Podemos negar a la parrbèsía filodemea toda dimensión específicamente epicúrea? Desde ya, la teorización epicúrea acerca de la dinámica de la parrhèsía no representa un quiebre absoluto respecto de la tradición: por un lado, a pesar de los recaudos que expresaré más abajo al respecto, la propuesta de Giovacchini de entender el PP a la luz de los tratados médicos hipocráticos quizás no debe ser desatendida por completo, en función de los plausibles paralelos que existen en cuanto a la estructuración de las reglas que gobiernan la relación médico/paciente y maestro/alumno. Por otra parte, la vinculación de la parrhésía con la kolakeia, en el marco de la definición de las virtudes que dan cuerpo a la verdadera philía, efectivamente resurge, aunque más no sea de modo ocasional, en el tratado, inscribiéndolo en una tradición de muy larga data. Sin embargo, junto ambos elementos, que pueden ser considerados como signos de relativa continuidad entre las prácticas epicúreas y su contexto cultural, emergen una serie de elementos que, hasta donde podemos constatar, representan innovaciones innegables, y que efectivamente otorgan a la parrhèsía epicúrea una dimensión pedagógica específica e inédita.

¿Cuáles son dichos elementos? El primero de ellos, que no ha sido tomado en consideración por Giovacchini, concierne al problema del beneficiario específico de la parrhèsía, el cual deviene, en el interior del PP, el alumno o, en términos más amplios, aquél que ha cometido un error y que se ha vuelto, por ello, objeto de instrucción y corrección. Que ése sea el beneficiario marca un quiebre importante respecto de lo que podemos reconstruir como la concepción pedagógica de la noción de parrhèsía (o, cuanto menos, de la forma predominante en la que la misma fue concebido durante el período clásico), dado que las especulaciones preepicúreas respecto de dicha noción tendían a poner el énfasis no el destinatario del discurso, sino en el sujeto que lo enunciaba, y, más específicamente, en el derecho del sujeto (libre) a enunciar un discurso de cierto tipo. El contexto ha mutado 
en forma sustantiva, por supuesto, y el reclamo respecto del derecho a la libre expresión no tiene lugar alguno en el seno de una comunidad filosófica — no al menos en el discurso de sus directivos (i.e., Zenón)—. La perspectiva pedagógica es, ahora, la que ocupa la escena, y la reflexión concierne al efecto específico del discurso parrhesiástico en el alma del que lo recibe, lo cual transforma al $P P$ en un tratado acerca de la eficacia de la parrhêsía como estrategia terapéutica ${ }^{22}$.

El segundo punto a tener en cuenta al evaluar la interpretación de Giovacchini se vincula con el problema acerca del borizonte deontológico que la autora sugiere como marco de interpretación del PP. De acuerdo a la autora, en efecto, una lectura detenida del tratado trae a la luz concordancias fundamentales entre los lineamientos filodemeos y las reglas deontológicas establecidas por la tradición hipocrática para regular la interacción paciente-médico. En paralelo con dicha tradición, Giovacchini sugiere pensar el PP desde la exhortación a tener en cuenta la singularidad del otro, sus deseos y sus derechos ${ }^{23}$, a fin de desterrar prácticas que desatienden al alumno en tanto sujeto no sólo físico sino también moral ${ }^{4}$.

A pesar de la plausibilidad inicial del enfoque propuesto por la autora, sin embargo, varias constataciones importantes se hacen evidentes, cuando nos volcamos al texto, que ponen en cuestión la adecuación de dicha perspectiva. La primera de ellas tiene que ver con el hecho de que el PP ofrece (independientemente del ordenamiento de fragmentos que adoptemos) un análisis relativamente sistemático de los tipos de disposiciones que podemos encontrar en el destinatario del discurso y, más importante aún, con el hecho de que dicho análisis está realizada con los ojos puestos en la efectividad pedagógica de la parrhèsía. Si bien es cierto, en este sentido, que la parrhèsía filodemea se construye estrictamente sobre la atención

\footnotetext{
${ }^{22}$ No deja de ser interesante, en este sentido, que este desplazamiento del centro de la parrhessía se va a ver reencauzado, ya en pleno período Imperial, en la Roma de Luciano de Samóstata, para quien la parrhèsía, como señala Holland, ya no tendrá por objetivo el cuidado del otro, sino la denuncia abierta de la hipocresía de los poderosos: "It is no longer necessary that parrhèsía be directed to those it is theoretically intended to benefit. Lucian did not read his satires before gatherings of philosophers, but in front of educated audiences seeking entertainment. His frank criticism is not intended to correct privately an erring fellow philosopher, but rather to ridicule publicly those who fail to live up to the standards of the great philosophers they claim to follow. This sort of parrhesía is not intended to foster the moral growth of the frauds whom he publicly and repeatedly denigrates and dismisses as rogues and cheats. He wants to expose these charlatans, not reform them." (Holland 263).

${ }^{23}$ Cf. Giovacchini 310.

${ }^{24}$ Cf. Giovacchini 308.
} 
de la singularidad del alumno, como afirma Giovacchini, nada de ello se debe a una preocupación por resguardar sus derechos, sino simplemente a la necesidad de definir las condiciones específicas que harán que la parrbèsía sea efectiva ante cada tipo de alumno (mujeres, viejos, jóvenes soberbios, iracundos, etc.).

Si contraponemos el enfoque filodemeo al hipocrático, obtenemos un panorámica clara de la enorme distancia que separa los intereses que guían la reflexión en cada uno de los dos enfoques. La literatura médica hipocrática, por un lado, se hallaba atravesada tanto por reflexiones relativas a la actitud correcta del médico como por estudios sistemáticos relativos a las distintas disposiciones (fisiológicas) de los pacientes; la primera perspectiva tenía por objetivo definir el horizonte deontológico de la práctica médica, la segunda respondía a la necesidad práctica de definir el mejor tratamiento posible, es decir, el más eficaz desde el punto de vista de la curación, y no el que mejor respetara los derechos y la dignidad del paciente. En el caso del $P P$, por el contrario, la reflexión de tipo práctico deviene central y exclusiva, en la medida en que tanto el análisis de la actitud del maestro como el de la disposición del alumno se hallan guiados por intereses prácticos. Desde esta perspectiva, parece plausible asumir que la discusión que parece haber estructurado el PP (o, más bien, las lecciones de Zenón de Sidón) no gira, como pretende Giovacchini, "en torno al carácter lícito" de la parrhèsía (Giovacchini, 2010, p. 309), sino en torno las condiciones bajo las cuales la parrhèsía puede volverse un instrumento pedagógicamente efectivo.

Que éste es un marco más adecuado de lectura del $P P$, y que, como consecuencia de ello, lo que la autora propone interpretar como reglas deontológicas debería ser interpretado como reglas de eficacia, se hace evidente por dos motivos adicionales: en primer lugar, mientras que las reglas deontológicas hipocráticas fueron formuladas para poner límites a ciertos excesos y abusos que tenían lugar en forma sistemática en la práctica médica cotidiana ${ }^{25}$, nada en el $P P$ parece sugerir que Filodemo haya enfrentado un escenario análogo en el caso de la parrhèsía; nada sugiere que el motor de la reflexión haya sido poner fin a prácticas abusivas o violentas que caracterizaban a la dinámica de la parrbésía, ya sea dentro o fuera de la comunidad epicúrea. Hacerlo, por otra parte, supondría conceder validez a un elemento adicional que Giovacchini atribuye a Filodemo, y que, como ya he sugerido, carece de apoyo textual, a saber, la preocupación por la dignidad moral del alumno y por sus derechos como persona. Los textos que se han conservado

\footnotetext{
${ }^{25} \mathrm{Al}$ respecto, cf., entre otros, Temkin \& Temkin 319-348; Nutton 72-86; Van der Eijk 101-118.
} 
habilitan, en este sentido, dos lecturas posibles: o suponemos que Filodemo simplemente omitió reflexionar acerca de los derechos del alumno en tanto persona moral (sin por ello negarlos abiertamente), o bien suponemos que no consideró que aquellos tuvieran derecho alguno en tanto alumnos, y la opción por una de las dos alternativas dependerá de cómo se interpreten ciertos pasajes claves del $P P$, referidos fundamentalmente a los insultos y los castigos físicos ${ }^{26}$. Pero sea cual sea la interpretación que asumamos (a saber, la de la mera desatención de los derechos del alumno o la de la negación de los mismos), lo decisivo es que ninguna de las dos opciones implica en absoluto conceder que Filodemo pueda haber hecho del respeto por el alumno un motor de su reflexión. La prohibición filodemea de intervenir con brusquedad, desprecio, etc., no tiene por objetivo, en este sentido, "atenuar la crueldad del hablar franco" (Giovacchini, 2010, p. 307), sino simplemente garantizar que la parrhèsía se desarrolle fluidamente, evitando que la vergüenza o la humillación obstaculicen las capacidades racionales del alumno para comprender aquello que el maestro está intentando poner antes sus ojos ${ }^{27}$.

Que la preocupación de Filodemo sea pedagógica y no ética, explica, incidentalmente, que dedique tanto tiempo a la determinación de las condiciones que definen una parrhèsía adecuada por parte del maestro como por parte del alumno. ¿Cuáles son esas condiciones? En líneas generales, podríamos resumirlas diciendo que para que la parrbésía sea exitosa, el alumno

a) debe ser consciente de que ha errado (Col. 15b);

b) debe estar abierto a la posibilidad de ser corregido (Cols. 16b-17b, 18b);

c) debe reconocer al maestro como alguien que

${ }^{26}$ Cf., vg., Frs. 21 y 83. Para una lectura no demasiado caritativa de las prácticas epicúreas, vid. Nussbaum 1993.

${ }^{27}$ Esta conclusión puede, a primera vista, parecer inconsistente en relación con el primer elemento que señalaba más arriba en contra de la lectura de Giovacchini, en la medida en que aquél enfatizaba el hecho de que, en el interior del PP, es el destinatario de la parrhésía (i.e., el alumno) quien deviene el centro de atención, mientras que esta última conclusión niega toda preocupación por la dignidad del otro (del alumno) en cuanto sujeto de derechos. La contradicción es meramente aparente, no obstante, dado que bien puedo poner el cuidado del otro como objeto de mi preocupación, sin reconocerle derecho alguno. La actitud paternalista en la pedagogía clásica, después de todo, parece haber alcanzado su punto más radical durante el período helenístico (específicamente de mano del estoicismo), y suponer que el epicureísmo podría haber asumido una posición completamente contraria a la tradición pedagógica grecorromana requeriría una fundamentación para la que dudo que podamos encontrar apoyo textual. 
c.1) actúa por buena voluntad —en lugar de por motivos egoístas$\left(22 \mathrm{a}-24^{a}\right)$

c.2) y que es más sabio que él (Cols. 21a, 24a), fundamentalmente en cuanto a lo conveniente en relación con los asuntos de la vida real (Col. 20a).

El maestro, por su parte,

d) debe practicar la parrhèsía con empatía (Fr. 79);

e) no debe insultar ni ridiculizar al alumno (Fr. 79), ni asumir una actitud altiva, insolente o despreciativa (Fr. 37-38);

f) no debe realizar una crítica global del alumno, sino circunscribir la crítica al error específico que se quiere abordar (Fr. 78);

g) no debe intervenir al alumno en presencia de todos, sino cuando se halla solo o frente a un grupo reducido de compañeros (Fr. 82).

Si el objetivo del tratado consistiera en reglar desde el punto de vista ético las condiciones bajo las que se produce el acto de la parrbèsía, sería necesario explicar la insistencia por parte de Filodemo sobre a-e, es decir, sobre las condiciones que deben cumplirse de parte del alumno para que la parrhèsía sea exitosa. Si interpretamos $a-g$, por el contrario, como reglas de eficacia, la articulación con el resto del tratado se vuelve natural, en la medida en que se dichas reglas se articulan con, por ejemplo, el análisis de los distintos tipos posibles de intervención parrhesiástica $^{28}$, la distinción entre los distintos factores que explican porqué ciertos sujetos son más permeables a la intervención parrhesiástica que otros ${ }^{29}$, o la reflexión acerca de las razones por las cuales el maestro debería esperar hasta que la pasión se haya aplacado en el alumno antes de intervenir con su discurso ${ }^{30}$. Lo que vincula todos estos momentos, insisto, es la preocupación central que, según creo evidente, recorre la totalidad del tratado, a saber, la definición de las condiciones bajo las cuales la parrhèsía puede representar una herramienta pedagógica efectiva.

Si esto es así, en suma, creo que podemos conceder sin dificultades que el PP se halla estructurado alrededor de una reflexión que no tiene por objetivo definir las formas correctas o incorrectas de practicar la parrhèsía, sino las formas efectivas e inefectivas de hacerlo, una práctica cuyo éxito o fracaso se medirá no por el grado

\footnotetext{
${ }^{28}$ Cf., vg., Fr. 6, 45; Col. 5b.

${ }^{29}$ Cf., vg., Cols. 22a-24a.

${ }^{30}$ Cf., vg. Fr. 65, 66.
} 
en el cual el sujeto que interviene pueda lograr ejercer su derecho a expresarse con franqueza, sino por el grado en el cual haya contribuido a instruir, corregir o "salvar" al alumno. Podemos ir un paso más allá y remarcar que estamos ante una práctica no solo especificamente pedagógica, sino también escolar, en la medida en que se trata de una práctica pensada para ser desplegada en el interior de la comunidad y sobre la base de la interacción (a la vez jerárquica y flexible) entre maestro y alumno ${ }^{31}$.

Ahora bien: que constituya una práctica especificamente pedagógica y que sea analizada desde una perspectiva especificamente escolar no significa que necesariamente represente una práctica especificamente epicúrea, dado que bien podría ser el caso de que otras escuelas del período hayan estructurado sus propias prácticas parrhesiásticas sobre una matriz pedagógica, y que Epicuro, o alguno de sus discípulos, haya tomado prestado de allí dichas prácticas y las haya incorporado al repertorio terapéutico epicúreo. ¿Podemos apelar, a fin de resolver este problema, a la presencia entre los fragmentos del PP de discusiones de temáticas no solo específicamente filosóficas, sino también específicamente epicúreas ${ }^{32}$ e interpretar dicha presencia como apoyo para la hipótesis de una parrhèsía específicamente epicúrea? Creo que es evidente que no, en la medida en que dichas doctrinas no aparecen entretejidas en la dinámica técnica de la parrbèsía, sino que aparecen simplemente como objetos del discurso parrhesiástico, un discurso que podría ser enfocado, en principio, a cualquier principio filosófico. No hay razón alguna, después de todo, para descartar la posibilidad de que la práctica de la parrbésía analizada por Filodemo describa adecuadamente una práctica que también los estoicos, por ejemplo, desarrollaban en el interior de su escuela, enfocándola, por ejemplo, sobre las doctrinas de los indiferentes o de la suficiencia de la virtud para la eudaimonia. Dada la matriz intelectualista que subyace a la teoría de la acción defendida por cada una de las dos escuelas, no sería implausible pensar, en este sentido, que la parrbèsía filodemea se adecuaría a la perfección a la terapia de tipo cognitivo desarrollada por el estoicismo, tanto antiguo como romano. No hay evidencia alguna, es cierto, de que algo así haya sucedido, y el silencio respecto de la utilización pedagógica de la parrhèsía en otras escuelas del período helenístico

\footnotetext{
${ }^{31}$ Cabe destacar, en este sentido, el hecho de que sólo un pequeñísimo puñado de fragmentos del PP puede ser leído como referencias a la práctica de la parrhésía con individuos externos a la comunidad epicúrea (Cols. 10b, 23b, 24a), mientras que el resto se apoyar claramente en la relación maestro-alumno.

${ }^{32}$ Cf., a modo de ejemplo, Col. 14a.
} 
es absoluto. Deducir a partir de dicho silencio, no obstante, que la utilización pedagógica de la parrbēsía representó una innovación epicúrea, representa una clara falacia. Todo lo que podemos decir es, a modo de síntesis, que — sin certeza alguna respecto de qué es lo que sucedía al mismo tiempo en otras escuelas o comunidades filosóficas - el epicureísmo, quizás desde las primeras generaciones posteriores a Epicuro, hizo un uso específicamente pedagógico-terapéutico de la parrhèsía y que, llegado el siglo I a.C., dicha práctica fue objeto de una reflexión teórica profunda y sistemática de la mano de Zenón de Sidón, reflexión que fue preservada por escrito en el tratado de Filodemo de Gadara Peri parrbēsias.

\section{CONCLUSIÓN}

¿Es posible, después de lo visto hasta el momento, retornar a la interpretación pedagógica de la parrbèsía epicúrea? En algunos aspectos, claramente no: la precisión del análisis de Giovacchini establece, como tarea todavía pendiente, la revisión de cuanto menos tres elementos que buena parte de la interpretación pedagógica había considerado como constitutivos de la parrbèsía: su carácter rotativo, la práctica de la delación amigable y la caracterización de la parrbēsía como un técnica estocástica. En cuanto al primero, se hace evidente la necesidad de realizar una reconstrucción más precisa del carácter rotativo de la parrhèsía, en lugar de asumir una dinámica absolutamente libre en el intercambio de lugares entre maestro y alumno. La figura de la "delación amigable", por su parte, puede haber sido dotada por ciertos defensores de la interpretación pedagógica de un lugar desproporcionadamente central en la dinámica comunitaria, y una revisión de la verdadera relevancia de los fragmentos preservados se vuelve también perentoria, de modo similar a lo que sucede con el carácter estocástico de la parrhêsía como técnica psicagógica, el cual parece haber recibido un tratamiento desmesurado, si consideramos el escaso apoyo textual que la idea de una técnica conjetural ofrece el PP.

A pesar de ello, y por las razones que he esgrimido en la sección anterior, considero que el núcleo central de la interpretación pedagógica puede seguir siendo defendida como una alternativa sumamente sólida y, fundamentalmente, como la única que, hasta el momento, permite traer a la luz la riqueza del PP desde el punto de vista de su aporte para la historia de la pedagogía y para nuestra comprensión de la dinámica interna de la comunidad epicúrea. Una comprensión profunda de la parrhèsía epicúrea implicaría, desde ya, analizar en profundidad y en forma sistemática los mecanismos mediante los cuales los epicúreos suponían 
que la parrhèsía operaba: ¿es la generación de sentimientos como la vergüenza, el miedo o la culpa la que garantiza la efectividad de la intervención parrhesiástica como práctica terapéutica? ¿ $\mathrm{O}$ dicha efectividad depende, por el contrario, de procesos puramente cognitivos? ¿Cómo se articula la parrhèsía con las prácticas de memorización estimuladas por la escuela desde sus inicios? Una relectura del PP a la luz de este tipo de interrogantes, lo cual excede el objetivo de estas páginas, nos pondría en una posición más favorable para construir una imagen de conjunto más acabada de la dinámica pedagógica de las comunidades epicúreas, al menos en tiempos de Zenón y Filodemo.

Rodrigo Braicovich Consejo Nacional de Investigaciones Cientificas y Tecnológicas (CONICET) Universidad Nacional de Rosario rbraicovich@gmail.com

\section{BIBLIOGRAFÍA}

Asmis, E. (2001): "Basic education in Epicureanism”, en Y.L. Too (ed.), Education in Greek and Roman Antiquity, Leiden: Brill, pp. 209-239.

Braicovich, R. (2014): "On the notion of ethical exercises in Epictetus", Prometeus, n'15, pp. $125-138$.

DeLATTRE, D. (2015): “La pratique maîtrisée du franc-parler: Philodème de Gadara, Le franc-parler (col. 151-162 D.), en M. Loubet \& D. Pralon (eds.), Poïkilö̈ karpoï: exégèses païennes, juives et chrétiennes, Aix-en-Provence: Presses Universitaires de Provence, pp. 435-453.

DE WITT, N. W. (1936): "Organization and procedure in Epicurean groups", Classical Philology, no 31(3), pp. 205-211.

- (1937): "The later paideia of Epicurus", Transactions and Proceedings of the American Philological Association, no 68, pp. 326-333.

- (1954): Epicurus and his Philosophy, Minneapolis: University of Minnesota Press.

ERLER, M. (2011a): "Autodidact and student: on the relationship of authority and autonomy in Epicurus and the Epicurean tradition", en J. Fish \& K. R. Sanders (eds.), Epicurus and the Epicurean Tradition, Cambridge: Cambridge University Press, pp. 9-28.

- (2011b): "Parrhesy and irony: Plato's Socrates and the Epicurean tradition", en R.A.H. King \& D.R. Schilling (eds.), How Should One Live?: Comparing Ethics in Ancient China and Greco-Roman Antiquity, Berlin: De Gruyter, pp. 155-169.

FERNANDES, E. (2009): “Tradição e atualidade da parrêsia («fala franca») como terapia”, en M. C. Diniz Peixoto (ed.), A saúde dos antigos. Reflexões gregas e romanas, Sao Paulo: Loyola, pp. 163-179. 
Foucault, M. (1994): Histoire de la sexualité. 3: Le souci de soi, París: Gallimard.

- (2001): L’herméneutique du sujet: cours au Collège de France, 1981-1982, París: Gallimard.

GaINES, R. N. (2004): "Cicero, Philodemus, and the development of Late Hellenistic rhetorical theory”, en J. T. Fitzgerald, D. Obbink, \& G. S. Holland (eds.), Philodemus and the New Testament World, Leiden: Brill, pp. 197-220.

Gigante, M. (1975): “Philosophia medicans”, Cronache Ercolanesi, no 5, pp. 53-61.

- (1983): Ricerche filodemee, Napoli: G. Macchiaroli.

Giovacchini, J. (2010): "La nouvelle reconstruction du rouleau du Franc-parler de Philodème permet-elle encore de postulerl'existence d'une parrhesia spécifiquement épicurienne?", en A. Antoni, G. Arrighetti, M.I. Bertagna, \& D. Delattre (eds.), Miscellanea Papyrologica Herculanensia, Pisa: Fabrizio Serra, pp. 293-314.

GLAD, C. E. (1996): "Frank speech, flattery and friendship in Philodemus", en J. T. Fitzgerald (ed.), Friendship, Flattery, and Frankness of Speech. Studies on Friendship in the New Testament World, Leiden: Brill, pp. 7-19.

- (2010): Paul and Philodemus. Adaptability in Epicurean and early Christian Psychagogy, Atlanta: Society of Biblical Literature.

Holland, G. S. (2004): “Call Me Frank: Lucian's (Self-)Defense of Frank Speaking and Philodemus' Peri Parrhesias”, en J. T. Fitzgerald, D. Obbink, \& G. S. Holland (eds.), Philodemus and the New Testament World, Leiden: Brill, pp. 245-269.

KEMP, J. (2010): "Flattery and frankness in Horace and Philodemus". Greece and Rome, no $57(1)$, pp. 65.

KOnSTAN, D. (1996): "Friendship, frankness and flattery", en J. T. Fitzgerald (ed.), Friendship, Flattery, and Frankness of Speech. Studies on Friendship in the New Testament World, Leiden: Brill, pp. 7-19.

—, Clay, D., Glad, C. E., Thom, J. C., \& WArE, J. (2007). "Introduction”, en Philodemus, On Frank Criticism, Atlanta: Society of Biblical Literature, pp. 1-24.

Momigliano, A. (1973): "Freedom of speech in Antiquity", en P.P. Wiener (ed.), Dictionary of the History of Ideas. Studies of Selected Pivotal Ideas, Nueva York: Scribner, vol. 2, pp. 252-263.

Nussbaum, M. (1993): “Argumentos terapéuticos: Epicuro y Aristóteles”, en M. Schofield \& G. Striker (eds.), Las normas de la naturaleza, Buenos Aires: Manantial, pp. 41-83.

- (1996): The Therapy of Desire. Theory and Praxis in Hellenistic Ethics, Nueva Jersey:

Princeton University Press.

Nutton, V. (2004): Ancient Medicine, London: Routledge.

Olivieri, A, (1914): Philodemi Peri Parrhesias Libellus, Leipzig: Teubner.

Philodemus (2007): Peri Parrhèsías] On Frank Criticism, D. Konstan, D. Clay, C. E. Glad, J. C. Thom, \& J. Ware (Trads.), Atlanta: Society of Biblical Literature.

SIDER, D. (2004): “How to commit philosophy obliquely: Philodemus' Epigrams in the light of his Peri Parrhesias", en J. T. Fitzgerald, D. Obbink, \& G. S. Holland (eds.), Philodemus and the New Testament World, Leiden: Brill, pp. 85-101. 
Temkin, O., \& Temkin, C. L. (eds.). (1967): Ancient Medicine. Selected Papers of Ludwig Edelstein, Baltimore: Johns Hopkins Press.

TsounA, V. (2007): The Ethics of Philodemus, Oxford: Oxford University Press.

- (2009): "Epicurean therapeutic strategies", en J. Warren (ed.), The Cambridge Companion to Epicureanism, Cambridge: Cambridge University Press, pp. 249-265.

VAN DER EIJK, P. (2005): Medicine and Philosophy in Classical Antiquity. Doctors and Philosophers on Nature, Soul, Health and Disease. Cambridge, Cambridge University Press.

White, L. M. (2004): "A measure of Parrhesia: The state of the manuscript of PHerc. 1471”, en J. T. Fitzgerald, D. Obbink, \& G. S. Holland (eds.), Philodemus and the New Testament World, Leiden: Brill, pp. 85-101. 